\title{
Energy-Efficient Spatial Modulation in Massive MIMO Systems by Means of Compressive Sensing
}

\author{
Adrian Garcia-Rodriguez and Christos Masouros \\ University College London (UCL), London, UK \\ Email: adrian.rodriguez.12@ucl.ac.uk
}

\begin{abstract}
In this paper we propose a spatial modulation (SM) technique with improved energy efficiency (EE) for the multiple access channel (MAC) with a large number of antennas. The proposed scheme builds upon compressive sensing (CS) and accounts for the sparsity and structure of the signals transmitted via SM in multi-user scenarios to further improve the performance and reduce the complexity of linear detectors. In particular, the proposed technique incorporates additional prior knowledge to conventional CS-based approaches by exploiting the existence of a maximum number of active antennas per user when SM transmission is used in the MAC. The results presented in this paper show that the proposed algorithm offers both a) reduced complexity and b) improved performance compared to conventional CS and linear detection strategies and also allow us to determine the conditions under which the use of SM systems in the MAC is beneficial from an EE point of view.

Index Terms-Spatial modulation, large-scale MIMO, multiple access, compressive sensing, energy efficiency.
\end{abstract}

\section{INTRODUCTION}

The development of wireless communication strategies to satisfy the increasingly higher data rate specifications has been recently focused on the optimization of a metric that combines both the spectral efficiency and the total power consumption: the energy efficiency [1], [2]. These are the cases of massive multiple-input multiple-output (MIMO) and SM systems, which exploit the benefits of increasing the number of antennas in the communication [3], [4]. In particular, massive MIMO proposes to comprise a large amount of antennas at the base stations (BSs) to facilitate the use of conventional linear precoding and detection schemes, which become optimal in the large-scale limit [3]-[5]. However, the high number of radio frequency $(\mathrm{RF})$ chains necessary in these systems can harm the EE [6]. For this reason, SM has been posed as an alternative in which only a subset of antennas are simultaneously active and the transmit antenna indexes are used as an additional source of information [7]. This, in turn, penalizes the achievable rates with respect to conventional spatial multiplexing techniques and modifies the operation at the receiver because the antennas used for transmission must also be determined [7].

Numerous works have focused on the development of lowcomplexity detectors and precoders for peer-to-peer (P2P) SM systems (see, e.g., [8]-[11] and references therein). This is the case of [11], where a normalized CS detection algorithm for space shift keying (SSK) and generalized space shift keying (GSSK) systems was proposed. The fundamental idea behind the approach of [11] is based on exploiting that GSSK systems only encode information in the active antenna locations and, therefore, a normalization of the channel matrix before the application of greedy compressive detectors can improve performance. However, the authors of [11] restrict their study to SSK and GSSK systems and do not account for the structured sparsity of the signals transmitted via SM in the MAC [7], [12].

The possibility of using SM mobile stations (MSs) in the MAC has attracted considerably less attention although theoretical [13], [14], and more practical studies [15] have shown its potential [7]. Specifically, two detection algorithms for the scenarios considered in this paper are proposed in [15]: a message passing detection (MPD) algorithm, and a more complex local search detection. This paper shows that SM systems can outperform conventional spatially multiplexed systems with the same spectral efficiency. However, the total number of operations of the MPD algorithm is conditioned by the number of messages conveyed between the nodes, which is inherently high if a massive base station (BS) is used [16]. In fact, this algorithm can also be used to obtain sparse reconstructions in $\mathrm{CS}$, albeit with a higher complexity than most state-of-the-art iterative reconstruction algorithms [16].

Instead, in this paper we focus on offering a low-complexity alternative based on CS specifically tailored for massive SM systems in the MAC. Particularly, we exploit the fact that a small number of antennas are simultaneously active (sparsity) and that each user can only activate a given number of antennas determined by the available RF chains (structure) [7]. This allows us to improve the performance and convergence speed of CS-based algorithms [12]. Moreover, as opposed to the conventional approaches, here we study the benefits of using CS-based detection schemes in overdetermined systems with a large number of receive antennas. We do this by relating the exploitation of the signal sparsity with the massive MIMO theory already available for linear detectors [3].

At this point, we remark that the proposed concept can also be applied to more complex algorithms for sparse signal reconstruction such as the ones based on MPD [12], [16]. However, in the following we concentrate on low-complexity greedy CS algorithms since the large system dimensions make their use more convenient from a complexity standpoint [11]. Additionally, the comparison of SM systems with regular spatially multiplexed transmission in the massive MAC allows us to characterize the improvements of the proposed scheme and determine the conditions under which SM transmission is more energy efficient than conventional MIMO. 


\section{A. Notation}

The superscripts $(\cdot)^{T},(\cdot)^{H}$ and $(\cdot)^{\dagger}$ denote transpose, conjugate transpose and pseudoinverse respectively. $\mathbf{I}_{M}$ represents the $M \times M$ identity matrix and $\|\cdot\|_{p}$ represents the $\ell_{p}$ norm. $\mathbb{E}[\cdot]$ is the expectation operator and $\sim$ reads as "distributed as". $\left.\mathbf{h}\right|_{\mathcal{H}}$ denotes the entries of the vector given by the indexes of the set $\mathcal{H}, \mathbf{H}_{\mathcal{H}}$ is the submatrix obtained by selecting the columns of $\mathbf{H}$ determined by $\mathcal{H}$, and $\operatorname{supp}(\cdot)$ represents the indexes of the non-zero entries. Moreover, $|\cdot|$ denotes the cardinality of a set, $\subset$ represents a subset and $\otimes$ denotes the Kronecker product.

\section{SySTEM Model}

\section{A. Multiple Access Channel (MAC)}

The general model considered hereafter is a MAC comprised of a single BS with $N$ receive antennas and $K \ll N$ MSs with $n_{t}$ transmit antennas per device. Let $M=K \cdot n_{t}$ denote the total number of antennas allocated at the MSs. In this setting, the signal received by the BS is given by

$$
\mathbf{y}=\mathbf{H x}+\mathbf{w},
$$

where $\mathbf{x} \in \mathbb{C}^{M \times 1}$ represents the signal conveyed by the MSs, $\mathbf{y} \in \mathbb{C}^{N \times 1}$ is the received signal, and $\mathbf{w} \in \mathbb{C}^{N \times 1} \sim$ $\mathcal{C N}\left(0, \sigma_{n}^{2} \mathbf{I}_{N}\right)$ is the additive white Gaussian noise with variance $\sigma_{n}^{2}$. Here, $\mathbf{H} \in \mathbb{C}^{N \times M} \sim \mathcal{C N}\left(\mathbf{0}, \mathbf{I}_{N} \otimes \mathbf{I}_{M}\right)$ characterizes the channel matrix of a frequency flat channel over Rayleigh fading, which can represent a subcarrier if orthogonal frequency division multiplexing (OFDM) is used in frequencyselective channels.

The transmit signal-to-noise ratio (SNR) of the MAC depends on the number of antennas simultaneously active at the MSs, $N_{a} \leq M$, and the transmission power. Hence, considering that $\mathbb{E}\left[x_{i} x_{i}^{H}\right]=1$ holds for the active antennas, the total transmit SNR can be expressed as

$$
\rho=\frac{\mathbb{E}\left[\mathbf{x}^{H} \mathbf{x}\right]}{\sigma_{n}^{2}}=\frac{N_{a}}{\sigma_{n}^{2}} .
$$

\section{B. Spatial Modulation in the Massive MAC}

To increase the uplink rates without significantly complicating the hardware at the mobile stations, SM proposes to activate a reduced number of antennas per transmitter and encode information into the spatial position of the active antennas [7]. In the following we consider that MSs only activate a single antenna for brevity, although extension to transmitters with a higher number of active antennas is straightforward. In SM transmission, each MS determines the antenna to activate and the constellation symbol based on the input bit stream [7]. Formally, the single-RF SM signal conveyed by the $k$-th user reads as

$$
\mathbf{x}_{k}=\left[0, \ldots, s_{p}^{q}, \ldots, 0\right]^{T},
$$

where $p \in\left[1, n_{t}\right]$ is the index of the active antenna and $s^{q}$ represents the $q$-th symbol of the transmit constellation $\mathcal{Q}$, with $|\mathcal{Q}|=Q$ being the modulation order. Here we note that the joint transmit signal $\mathbf{x}=\left[\mathbf{x}_{1}^{T}, \ldots, \mathbf{x}_{K}^{T}\right]^{T}$ is sparse, i.e., there are only a few non-zero components. We also remark that the hardware reduction experienced by SM also entails a decrease of the achievable rates when compared to conventional spatially multiplexed MIMO transmission [7]. For instance, while $S_{\mathrm{MIMO}}=K \cdot n_{t} \cdot \log _{2}(Q)$ bits per channel use can be simultaneously conveyed with regular MIMO transmission, SM is only able to convey $S_{\mathrm{SM}}=K\left(\log _{2}\left(n_{t}\right)+\log _{2}(Q)\right)$ bits when a single antenna is active per MS.

At the reception side, the BS estimates the conveyed constellation symbols and the antennas activated during uplink transmission [7]. However, the optimal maximum likelihood detector cannot be applied in systems with a high number of users due to its intractable complexity [8], [9]. Instead, the performance and low complexity of linear detection in the massive MAC makes its use appropriate in this setting [3]. The signal obtained after linear detection can be expressed as

$$
\mathbf{g}=\mathbf{D}(\mathbf{H} \mathbf{x}+\mathbf{w}),
$$

where $\mathbf{D} \in \mathbb{C}^{M \times N}$ is a linear detection matrix given by

$$
\mathbf{D}=\left(\mathbf{H}^{H} \mathbf{H}+\varsigma_{M}\right)^{-1} \mathbf{H}^{H} .
$$

Here, $\varsigma=0$ and $\varsigma=M / \rho$ for the zero forcing (ZF) and minimum mean square error (MMSE) detectors respectively. The transmitted constellation symbol $\hat{q}$ and the active antenna index $\hat{p}$ of the $k$-th user can be then obtained from (4) following

$$
\begin{gathered}
\hat{p}=\arg \max _{p}\left|g_{p}^{\{k\}}\right|, \\
\hat{q}=\mathcal{P}\left(g_{\hat{p}}^{\{k\}}\right) .
\end{gathered}
$$

Here, $g_{\{p, \hat{p}\}}^{\{k\}}$ denotes the $\{p, \hat{p}\}$-th entry of the decision vector $\mathbf{g}^{\{k\}}$ for the $k$-th user and $\mathcal{P}$ is the demodulation function.

However, the use of SM transmission in the MAC conventionally increases the total number of antennas at the MSs with respect to conventional spatially multiplexed MIMO, hence degrading the average performance of the linear detectors due to the worse conditioning of $\mathbf{H}$. Accordingly, in this paper we propose an enhanced CS-based detector to exploit the fact that only $N_{a} \ll M$ antennas are active, together with the knowledge of their distribution in the MAC. In other words, we look at scenarios where $N \gg M$ does not necessarily hold but $N \gg N_{a}$ brings the massive MIMO effect thanks to the use of CS.

\section{Structured Compressive Sensing Detection: Spatial Modulation Matching Pursuit (SMMP)}

CS acquisition systems exploit the fact that the transmitted signal $\mathrm{x}$ is sparse to improve the detection performance [17]. In order to do so, the channel matrix $\mathbf{H}$ must satisfy the restricted isometry property of order $N_{a}$, which is defined as [17]

$$
\left(1-\delta_{N_{a}}\right) \leq \frac{\|\mathbf{H x}\|_{2}^{2}}{\|\mathbf{x}\|_{2}^{2}} \leq\left(1+\delta_{N_{a}}\right)
$$

where $\delta_{N_{a}} \in(0,1)$. Based on the above, the application of CSbased detection to SM communication systems is a convenient 
strategy to improve the performance of conventional detectors because only a few antennas are simultaneously active [11]. Moreover, the channel matrix that arises in rich scattering environments with Rayleigh fading satisfies the RIP with $\delta_{N_{a}}<0.1$ provided that $N \geq c N_{a} \log \left(M / N_{a}\right)$, with $c$ being a small constant [11], [17]. Under these conditions, an accurate reconstruction of $\mathrm{x}$ can be obtained by solving [17]

$$
\begin{gathered}
\operatorname{minimize}\|\mathbf{x}\|_{1} \\
\text { subject to }\|\mathbf{H x}-\mathbf{y}\|_{2} \leq \eta,
\end{gathered}
$$

where $\eta$ is a constant that bounds the noise power $\left(\|\mathbf{w}\|_{2} \leq \eta\right)$. Nevertheless, since the $\ell_{1}$ minimization is computationally expensive, a range of techniques that provide a trade-off between complexity and performance has been developed [12], [18]. This is the case of the Compressive Sampling Matching Pursuit (CoSaMP) greedy algorithm, which additionally provides error guarantees in the detection of noisy sparse signals [18].

However, the trivial application of CS algorithms to the MAC in SM systems is far from optimal since the resulting distribution of the active antennas may not have physical sense. This is because generic CS reconstruction algorithms do not provide any additional knowledge about the location of the non-zero entries [12], [18]. This could generate situations in which multiple active antennas were allocated to the same user, a circumstance that cannot occur when conventional SM transmission is used. To overcome this issue, in the following we study an approach based on the CoSaMP iteration that accounts for the particularities of SM in the MAC to avoid this kind of errors and further improve performance [12].

The proposed algorithm is referred to as spatial modulation matching pursuit (SMMP) and its pseudocode is shown in Algorithm 1. The algorithm works as follows: Firstly, SMMP identifies the entries with a largest error in the estimated received signal as in the conventional CoSaMP [18]. With this purpose, the residual $\mathbf{r} \in \mathbb{C}^{N \times 1}$ is defined as

$$
\mathbf{r} \triangleq \mathbf{y}-\mathbf{H} \tilde{\mathbf{x}}^{i}=\mathbf{H}\left(\mathbf{x}-\tilde{\mathbf{x}}^{i}\right)+\mathbf{w},
$$

where $\tilde{\mathbf{x}}^{i} \in \mathbb{C}^{M \times 1}$ is the approximation of $\mathbf{x}$ generated at the $i$-th iteration. Once the residual signal has been computed, the output of a matched filter (MF), $\mathbf{p} \in \mathbb{C}^{M \times 1}$, is used to determine the plausible active antennas as

$$
\mathbf{p}=\mathbf{H}^{H} \mathbf{r}
$$

At this point, the conventional CoSaMP algorithm simply selects the entries with highest power to form the set with the indexes of the plausible active antennas $\Omega$ with cardinality $|\Omega|=k \geq K$ [18]. Instead, here we exploit the knowledge that only a given number of antennas per user is simultaneously active to define $\Omega$ [12]. Specifically, $\omega \subset \Omega$ is defined as the set comprised of the $K$ largest entries of $\mathbf{p}$ satisfying the constraint that one antenna per user must be active. This definition ensures the selection of one active antenna per user at the output of the MF, hence reducing the errors in the active antenna identification. The remaining $k-K$ entries are selected as the ones with highest energy independently of the user distribution as in the conventional CoSaMP algorithm.

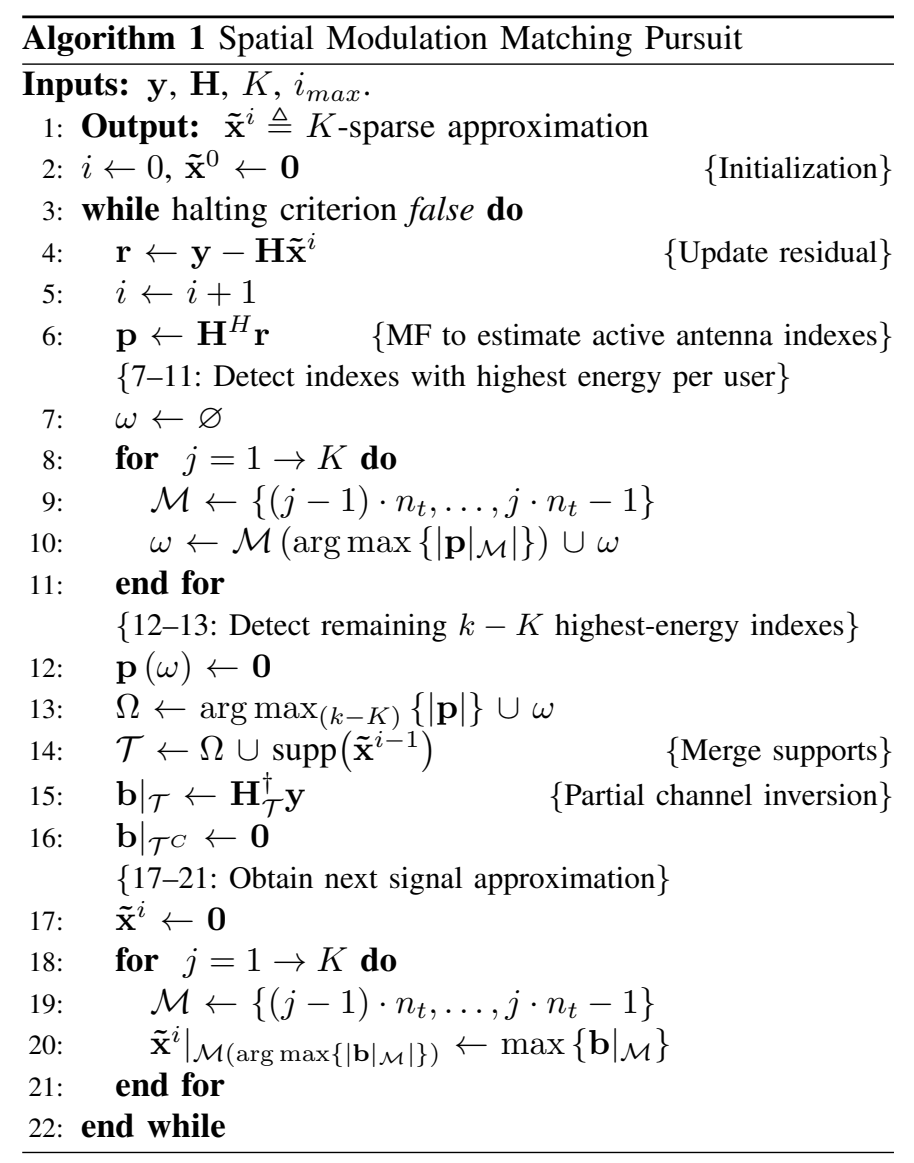

Once $\Omega$ is derived, the set $\mathcal{T}$ is defined as

$$
\mathcal{T} \triangleq \Omega \cup \operatorname{supp}\left(\tilde{\mathbf{x}}^{i-1}\right) \text {. }
$$

In plain words, $\mathcal{T}$ is the set built by combining the active antennas estimated during the previous iteration and other set that incorporates antennas that could also have been active but were not considered in $\tilde{\mathbf{x}}^{i-1}$ due to estimation errors. This set also determines the columns of the matrix $\mathbf{H}$ that will be used to solve the partial channel inversion

$$
\left.\mathbf{b}\right|_{\mathcal{T}}=\left(\mathbf{H}_{\mathcal{T}}^{H} \mathbf{H}_{\mathcal{T}}\right)^{-1} \mathbf{H}_{\mathcal{T}}^{H} \mathbf{y}=\left(\mathbf{H}_{\mathcal{T}}^{H} \mathbf{H}_{\mathcal{T}}\right)^{-1} \mathbf{H}_{\mathcal{T}}^{H}(\mathbf{H} \mathbf{x}+\mathbf{w}) .
$$

After this, $\tilde{\mathbf{x}}^{i}$ is built by selecting the largest entries of $\left.\mathbf{b}\right|_{\mathcal{T}}$ based on a user-by-user criterion and the next iteration is started. The algorithm finishes when the maximum number of iterations $i_{\max }$ is achieved or the norm of the residual is sufficiently small. Therefore, it can be seen that the active antenna identification steps 7-11 and 17-21 in Algorithm 1 have been modified w.r.t. the conventional CoSaMP to exploit the additional structure of SM in the MAC [12]. Moreover, these operations, although shown via iterative structures for simplicity, can be implemented via vector operations with reduced computational time. Overall, the proposed CS-based scheme can be interpreted as a hybrid detector in which the MF is used to determine the active antenna indexes and this knowledge is used to reduce the dimensions of the ZF detector to enhance its performance and reduce the global complexity.

At this point we would like to remark the implications 
of using a detection matrix with reduced dimensions in (13) when compared to (5). In particular, since the matrix inversion dominates the detection complexity with $\mathcal{O}\left(N M^{2}\right)$ [2], the dimensionality reduction ensures a low-complexity provided that the algorithm converges in a few iterations $i_{\max }$ as shown in Sec. VI. Additionally, since the relationship $N \gg|\mathcal{T}|$ conventionally holds in (13), the use of Algorithm 1 allows us to exploit massive MIMO benefits such as the robustness to noise or imperfect channel state information (CSI) offered by linear detection strategies. This enables us to further improve the performance of detection strategies that do not exploit the structured sparsity of SM transmission in the MAC [3], [12].

\section{Convergence Rate And ERror Analysis of CS-BASED DETECTION IN MASSIVE MIMO}

Although the error between $\mathbf{x}$ and its sparse approximation $\tilde{\mathbf{x}}^{i}$ was characterized in [18], its dependence on the number of users and receive antennas was not intuitive. For this reason, in this section we derive a more practical expression by exploiting the large dimensions of the systems considered in this paper.

Theorem 1: Let $\mathbf{H} \sim \mathcal{C N}\left(\mathbf{0}, \mathbf{I}_{N} \otimes \mathbf{I}_{M}\right)$. Then, the Euclidean norm of the error between the $K$-sparse signal generated at the $i$-th iteration of the generic and SMMP CS algorithms, $\tilde{\mathbf{x}}^{i}$, and the original transmitted signal, $\mathbf{x}$, is upper bounded by

$$
\left\|\mathbf{x}-\tilde{\mathbf{x}}^{i}\right\|_{2} \leq c_{1}(N, K)\left\|\mathbf{x}-\tilde{\mathbf{x}}^{i-1}\right\|_{2}+c_{2}(N, K)\|\mathbf{w}\|_{2} .
$$

In the above, generic CS refers to the conventional CoSaMP iteration without the proposed optimization and the functions $c_{1}(N, K)$ and $c_{2}(N, K)$ only depend on $K$ and $N$ and they are given by

$$
\begin{aligned}
c_{1} & =\left(2+\frac{\left(\frac{2+4 \sqrt{\beta(4 K)}}{\beta(4 K)}\right)}{\left(1-\frac{1}{\sqrt{\beta(3 K)}}\right)^{2}}\right) \frac{\left(\frac{1+2 \sqrt{\beta(4 K)}}{\beta(4 K)}\right)+\left(\frac{1+2 \sqrt{\beta(2 K)}}{\beta(2 K)}\right)}{\left(1-\frac{1}{\sqrt{\beta(2 K)}}\right)^{2}} \\
c_{2} & =\left(2+\frac{\left(\frac{2+4 \sqrt{\beta(4 K)}}{\beta(4 K)}\right)}{\left(1-\frac{1}{\sqrt{\beta(3 K)}}\right)^{2}}\right)\left(\frac{\left(2+\frac{2}{\sqrt{\beta(2 K)}}\right)}{\sqrt{N}\left(1-\frac{1}{\sqrt{\beta(2 K)}}\right)^{2}}\right) \\
+ & \frac{2}{\sqrt{N}\left(1-\frac{1}{\sqrt{\beta(3 K)}}\right)}
\end{aligned}
$$

where $\beta(V)=N / V$. Note that the above expressions are only valid when $N \geq 4 K$ holds. However, although not shown in this paper for brevity, similar expressions can be obtained for the more uncommon case of $N<4 K$.

Sketch of the proof. For reasons of space, in the following we only show a sketch of the proof. Specifically, to obtain (14) and (15) we use the results that characterize the maximum and minimum singular values of Wishart matrices with large dimensions [3]. In particular, let $\mathbf{W}=\mathbf{H}_{\mathcal{L}} \mathbf{H}_{\mathcal{L}}^{H}$ be a Wishart matrix with $\mathbf{H}_{\mathcal{L}} \in \mathcal{C}^{N \times|\mathcal{L}|}$ and satisfying $h_{m, n} \sim \mathcal{C N}(0,1)$. Then, for large $N$ and $|\mathcal{L}|<N$, the maximum and minimum

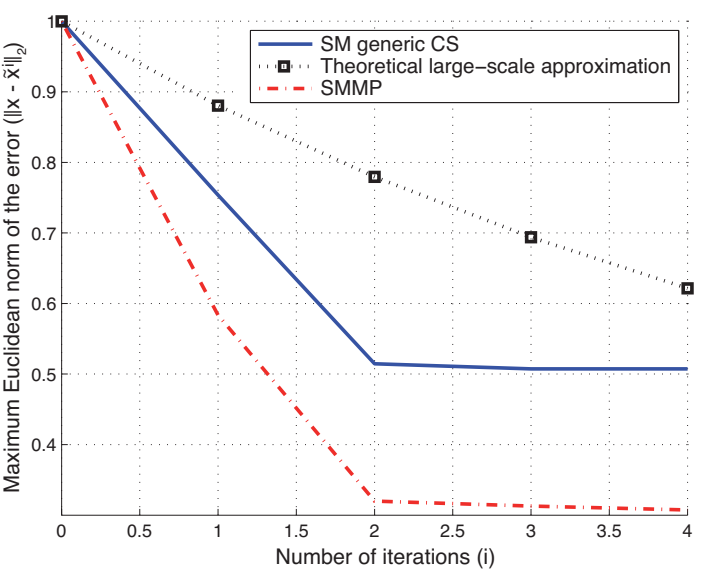

Fig. 1. Theoretical and empirical evolution of the maximum Euclidean norm of the error vs. number of iterations $(i)$ for $N=128, K=16, n_{t}=4$, $i_{\max }=4, k=2 K, 4$-QAM and $10^{5}$ channel realizations.

eigenvalues of $\mathbf{W}$ converge to [3]

$$
\begin{gathered}
\sigma_{\min }(\mathbf{W}) \longrightarrow N \times\left(1-\frac{1}{\sqrt{\beta(|\mathcal{L}|)}}\right)^{2} \\
\sigma_{\max }(\mathbf{W}) \longrightarrow N \times\left(1+\frac{1}{\sqrt{\beta(|\mathcal{L}|)}}\right)^{2}
\end{gathered}
$$

These expressions that apply to massive systems allow us to bound the Euclidean norm of the error at the $i$-th iteration by following similar arguments to the ones introduced in [18], but exploiting (16) and (17) where convenient.

Note that, as opposed to [18], (14) and (15) directly show the impact of $N$ and $K$ on the approximation error bound. This expression has been validated in Fig. 1, where the maximum empirical Euclidean norm of the error has been obtained over $10^{5}$ channel realizations for the proposed and the generic CS (CoSaMP) algorithms. The theoretical large-scale approximation (14) is shown for a number of receive antennas that ensures algorithmic convergence [18]. Fig. 1 allows us to derive two interesting conclusions regarding the behaviour of the greedy CS-based algorithms considered in this paper. In particular, it can be seen that the theoretical approximation provides a useful upper bound to characterize the evolution of the error, and that the proposed strategy offers a faster convergence and improved performance due to the reduced number of errors in the antenna detection stage.

\section{ENERGY EFFICIENCY}

The battery lifetime of the MSs is a critical parameter in the design of current wireless communication systems [1]. Therefore, since the power consumption of the transmission stage generally dominates the communication-related energy use [19], in this section we study the benefits that the proposed strategy offers in the EE. With this goal, we express the EE as the rate per milliwatt of total consumed power by using the metric [19], [20]

$$
\epsilon=\frac{S_{e}}{\sum_{k=1}^{K}\left(P_{C k}+P_{T k}\right)},
$$


where $S_{e}$ accounts for the spectral efficiency in bits per channel use (bpcu), $P_{T k}$ refers to the power consumption of the power amplifiers (PAs) of the $k$-th user, and $P_{C k}$ refers to the circuit power consumption of the rest of the electronic components required in the RF chains. $P_{T k}$ can be further decomposed as [20]

$$
P_{T k}=\zeta \cdot \sum_{j=1}^{n_{t}} p_{k, j}=\frac{\nu}{\eta} \cdot \sum_{j=1}^{n_{t}} p_{k, j},
$$

where $p_{k, j}$ denotes the power to be transmitted by the $j$-th antenna of the $k$-th user, and $\zeta=\frac{\nu}{\eta}$ is a coefficient that determines the total power consumption of the PA depending on the PA efficiency, $\eta$, and the peak to average power ratio (PAPR) of the modulation employed, $\nu$ [19]. Moreover, the power consumption of the remaining circuitry is given by [20]

$$
P_{C k}=P_{\Psi}+\sum_{j=1}^{n_{t}} P_{\psi, j},
$$

where $P_{\Psi}$ refers to the static power consumption, and $P_{\psi, j}$ corresponds to the power consumption of the circuitry and the digital signal processing that depends on the activation of the $j$-th antenna. Clearly, $P_{\psi, j}=0$ for the inactive antennas. The global EE can be therefore expressed as

$$
\epsilon=\frac{S_{e}}{\sum_{k=1}^{K}\left\{P_{\Psi}+\sum_{j=1}^{n_{t}} P_{\psi, j}+\frac{\nu}{\eta} \cdot \sum_{j=1}^{n_{t}} p_{k, j}\right\}},
$$

where, in the following, $P_{\Psi}$ is set to a reference of $5 \mathrm{~mW}$ per MS [20], and $\eta$ is considered to be 0.35 due to the linearity required to transmit QAM modulated signals [19].

\section{Simulation Results}

Numerical results have been obtained via Monte Carlo simulations to evaluate the performance and energy efficiency improvements of the proposed approach. The simulation setup is comprised of a BS with $N=128$ antennas, a Rayleigh fading channel as specified in Sec. II-A, and $K$ MSs. The modulation order $Q$ and the number of antennas per transmitter $n_{t}$ are set to ensure that the systems under comparison have the same spectral efficiency as conventionally considered in the literature [15], [21]. In this section, we compare the proposed CS-based detection schemes with different low-complexity strategies, namely the linear ZF and MMSE detectors described in (5), and conventional spatial multiplexing MIMO systems without SM.

Fig. 2 shows the bit error rate (BER) of the considered systems for increasing levels of SNR, imperfect CSI estimation and $K=48$. The estimated channel with imperfect CSI is modeled as $\hat{\mathbf{H}}=\sqrt{1-\tau^{2}} \mathbf{H}+\tau \mathbf{Z}$, where $\mathbf{Z} \in \mathbb{C}^{N \times M} \sim$ $\mathcal{C N}\left(\mathbf{0}, \mathbf{I}_{N} \otimes \mathbf{I}_{M}\right)$ is the uncorrelated channel estimation error and $\tau \in[0,1]$ is a parameter that determines the quality of the CSI estimation ranging from perfect $(\tau=0)$ to only statistical CSI $(\tau=1)$ [4]. In this figure, SM Generic CS denotes the conventional CoSaMP algorithm without exploiting the MAC structure, $\tau=0.1$, and the MSs in the SM systems incorporate $n_{t}=4$ antennas each. The results show that

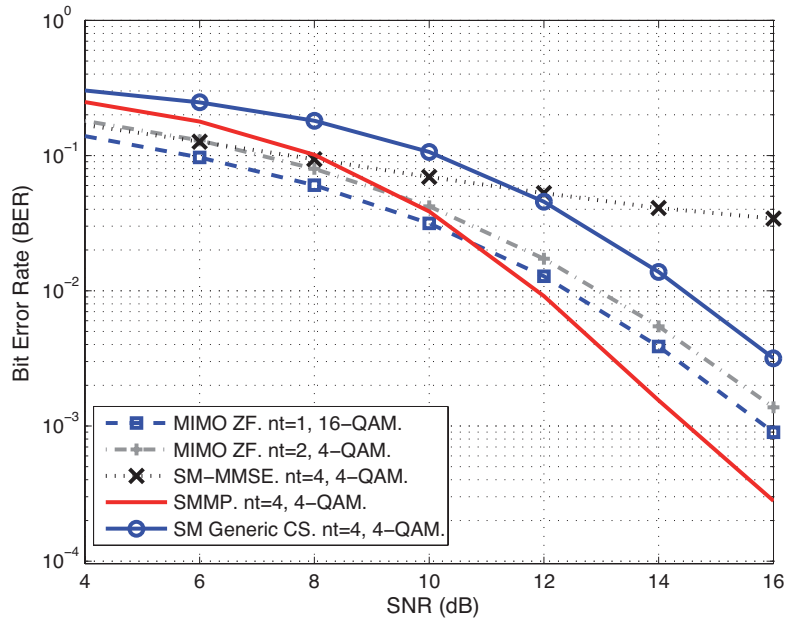

Fig. 2. BER vs. SNR for $N=128, K=48, i_{\max }=4, k=K, S_{e}=192$ bpcu and imperfect CSI $(\tau=0.1)$.

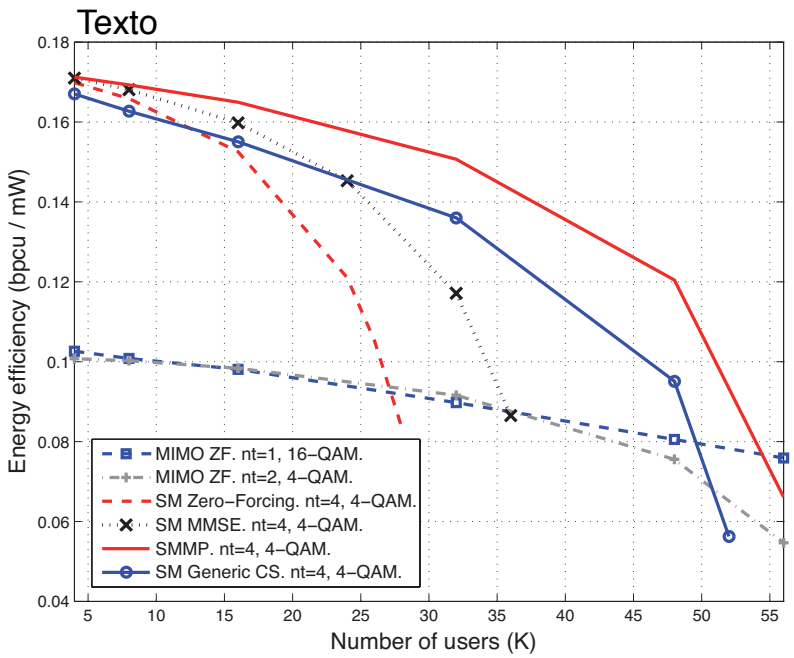

Fig. 3. EE vs. number of users $K$ to achieve BER $=10^{-3} \cdot N=128$ and $P_{\psi}=15 \mathrm{~mW}$ per active antenna.

the proposed strategy offers an improved performance w.r.t. the conventional alternatives for a large set of BERs. In particular, a performance gain higher than $1.5 \mathrm{~dB}$ to achieve an average BER of $10^{-3}$ can be observed when compared to the conventional MIMO system with a higher modulation order. We remark that this performance difference is maximized from an EE perspective since the PAPR of a transmitter with a higher modulation order is also increased, hence directly reducing the PA efficiency as shown in (21).

The above observation is explicitly shown in Fig. 3, where the energy efficiency of the systems under study is depicted for increasing number of users $K$. In this figure, the transmission power is varied depending on the number of users so that an average BER of $10^{-3}$ is guaranteed, and the noise variance is set to $\sigma_{n}^{2}=0.01$. Moreover, the circuit and signal processing power consumption that depends on the number of active antennas is fixed to $P_{\psi}=15 \mathrm{~mW}$ per active antenna in (20) [19]. From the results of this figure it can be concluded that SM systems outperform conventional MIMO systems for a small number of MSs whereas the latter ones are more useful 


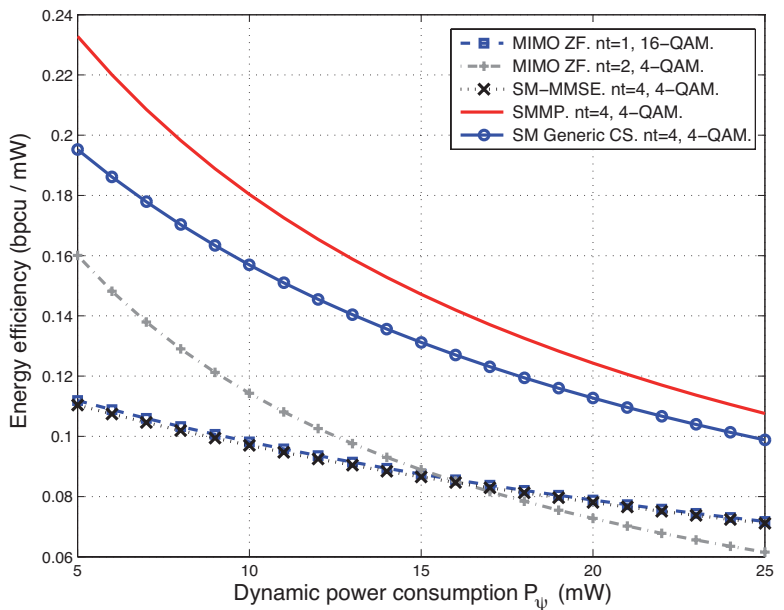

Fig. 4. EE vs. dynamic power consumption per active antenna $\left(P_{\psi}\right)$ to achieve $\mathrm{BER}=10^{-3} \cdot N=128, K=36$

when a large number of users are considered. Intuitively, this occurs because SM systems require allocating a higher number of antennas at the MSs to achieve the same spectral efficiency, hence reducing the system performance for high $K$ due to the poor conditioning of the channel matrix. Nevertheless, it can be seen that the proposed algorithm outperforms the rest of alternatives for a wide range of scenarios, hence validating the benefits of SM against conventional MIMO transmission in the MAC.

Fig. 4 shows the EE for increasing values of the dynamic power consumption per antenna $P_{\psi}$ and $K=36$. It can be seen that the use of SM is, in general, beneficial in this scenario. This is because the EE of a single-antenna MIMO is reduced due to the necessity of using a higher modulation order, which in this case increases the PAPR factor from 1 (QPSK) to 9/5 (16-QAM). Moreover, this figure also depicts that increasing the dynamic power consumption harms the EE of the system with conventional spatial multiplexing and two antennas per user due to the higher circuit power consumption required to activate the additional RF chain per user. Overall, these results characterize the improvements obtained by the exploitation of the MAC structure in CS-based algorithms.

\section{CONCLUSION}

In this paper, a CS-based detection algorithm that exploits the sparsity and particular signal structure of SM transmission in the MAC has been presented. The EE analysis of the MAC with a massive BS has exhibited that there exists a trade-off in the use of conventional MIMO transmission and SM, being one more useful than the other depending on parameters such as the number of users or the dynamic power consumption. Moreover, it has been shown that the proposed strategy is able to outperform conventional linear detectors, hence extending the benefits of SM in the MAC to a wider range of scenarios.

\section{ACKNOWLEDGMENT}

This work was supported by the Royal Academy of Engineering, UK and the EPSRC under grant EP/M014150/1.

\section{REFERENCES}

[1] G. Li, Z. Xu, C. Xiong, C. Yang, S. Zhang, Y. Chen, and S. Xu, "Energyefficient wireless communications: Tutorial, survey, and open issues," IEEE Wireless Communications, vol. 18, no. 6, pp. 28-35, 2011.

[2] E. Bjornson, L. Sanguinetti, J. Hoydis, and M. Debbah, "Optimal design of energy-efficient multi-user MIMO systems: Is massive MIMO the answer?" IEEE Trans. on Wireless Communications, vol. PP, no. 99, pp. $1-1,2015$.

[3] F. Rusek, D. Persson, B. K. Lau, E. Larsson, T. Marzetta, O. Edfors, and F. Tufvesson, "Scaling up MIMO: Opportunities and challenges with very large arrays," IEEE Signal Processing Magazine, vol. 30, no. 1, pp. 40-60, 2013.

[4] S. Wagner, R. Couillet, M. Debbah, and D. T. M. Slock, "Large system analysis of linear precoding in correlated MISO broadcast channels under limited feedback," IEEE Trans. on Information Theory, vol. 58, no. 7, pp. 4509-4537, 2012.

[5] C. Masouros, M. Sellathurai, and T. Ratnarajah, "Large-scale MIMO transmitters in fixed physical spaces: The effect of transmit correlation and mutual coupling," IEEE Trans. on Communications, vol. 61, no. 7, pp. 2794-2804, July 2013.

[6] D. Ha, K. Lee, and J. Kang, "Energy efficiency analysis with circuit power consumption in massive MIMO systems," in IEEE 24th International Symposium on Personal Indoor and Mobile Radio Communications (PIMRC), Sept 2013, pp. 938-942.

[7] M. Di Renzo, H. Haas, A. Ghrayeb, S. Sugiura, and L. Hanzo, "Spatial modulation for generalized MIMO: Challenges, opportunities, and implementation," Proceedings of the IEEE, vol. 102, no. 1, pp. 56-103, 2014.

[8] A. Younis, S. Sinanovic, M. Di Renzo, R. Mesleh, and H. Haas, "Generalised sphere decoding for spatial modulation," IEEE Trans. on Communications, vol. 61, no. 7, pp. 2805-2815, 2013.

[9] S. Sugiura, C. Xu, S. X. Ng, and L. Hanzo, "Reduced-complexity coherent versus non-coherent QAM-aided space-time shift keying," IEEE Trans. on Communications, vol. 59, no. 11, pp. 3090-3101, 2011.

[10] C. Masouros, "Improving the diversity of spatial modulation in MISO channels by phase alignment," IEEE Communications Letters, vol. 18, no. 5, pp. 729-732, May 2014.

[11] C.-M. Yu, S.-H. Hsieh, H.-W. Liang, C.-S. Lu, W.-H. Chung, S.-Y. Kuo, and S.-c. Pei, "Compressed sensing detector design for space shift keying in MIMO systems," IEEE Communications Letters, vol. 16, no. 10, pp. $1556-1559,2012$.

[12] R. G. Baraniuk, V. Cevher, M. F. Duarte, and C. Hegde, "Model-based compressive sensing," IEEE Trans. on Information Theory, vol. 56, no. 4, pp. 1982-2001, 2010.

[13] N. Serafimovski, S. Sinanović, M. Di Renzo, and H. Haas, "Multiple access spatial modulation," EURASIP Journal on Wireless Communications and Networking, vol. 2012, no. 1, pp. 1-20, 2012.

[14] M. Di Renzo and H. Haas, "Bit error probability of space-shift keying MIMO over multiple-access independent fading channels," IEEE Trans. on Vehicular Technology, vol. 60, no. 8, pp. 3694-3711, Oct 2011.

[15] T. Narasimhan, P. Raviteja, and A. Chockalingam, "Large-scale multiuser SM-MIMO versus massive MIMO," in Information Theory and Applications Workshop (ITA), Feb 2014, pp. 1-9.

[16] D. L. Donoho, A. Maleki, and A. Montanari, "Message-passing algorithms for compressed sensing," Proceedings of the National Academy of Sciences, vol. 106, no. 45, pp. 18 914-18 919, 2009.

[17] E. J. Candès and M. B. Wakin, "An introduction to compressive sampling," IEEE Signal Processing Magazine, vol. 25, no. 2, pp. 21-30, 2008.

[18] D. Needell and J. A. Tropp, "CoSaMP: Iterative signal recovery from incomplete and inaccurate samples," Applied and Computational Harmonic Analysis, vol. 26, no. 3, pp. 301-321, 2009.

[19] S. Cui, A. J. Goldsmith, and A. Bahai, "Energy-constrained modulation optimization," IEEE Trans. on Wireless Communications, vol. 4, no. 5, pp. 2349-2360, 2005.

[20] G. Miao, "Energy-efficient uplink multi-user MIMO," IEEE Trans. on Wireless Communications, vol. 12, no. 5, pp. 2302-2313, May 2013.

[21] K. Ntontin, M. Di Renzo, A. Perez-Neira, and C. Verikoukis, "Towards the performance and energy efficiency comparison of spatial modulation with conventional single-antenna transmission over generalized fading channels," in IEEE 17th International Workshop on Computer Aided Modeling and Design of Communication Links and Networks (CAMAD), 2012, pp. 120-124. 